\title{
Universal circles for quasigeodesic flows
}

\author{
DANNY CALEGARI
}

\begin{abstract}
We show that if $M$ is a hyperbolic 3-manifold which admits a quasigeodesic flow, then $\pi_{1}(M)$ acts faithfully on a universal circle by homeomorphisms, and preserves a pair of invariant laminations of this circle. As a corollary, we show that the Thurston norm can be characterized by quasigeodesic flows, thereby generalizing a theorem of Mosher, and we give the first example of a closed hyperbolic 3-manifold without a quasigeodesic flow, answering a long-standing question of Thurston.
\end{abstract}

57R30; 37C10, 37D40, 53C23, 57M50

\section{Introduction}

\subsection{Motivation and background}

Hyperbolic 3-manifolds can be studied from many different perspectives. A very fruitful perspective is to think of such manifolds as dynamical objects. For example, a very important class of hyperbolic 3-manifolds are those arising as mapping tori of pseudo-Anosov automorphisms of surfaces (Thurston [24]). Such mapping tori naturally come with a flow, the suspension flow of the automorphism. In the seminal paper [4], Cannon and Thurston showed that this suspension flow can be chosen to be quasigeodesic and pseudo-Anosov. Informally, a flow on a hyperbolic 3-manifold is quasigeodesic if the lifted flowlines in the universal cover are quasi-geodesics in $\mathbb{M}^{3}$, and a flow is pseudo-Anosov if it looks locally like a semi-branched cover of an Anosov flow. Of course, such a flow need not be transverse to a foliation by surfaces. See Thurston [24] and Fenley [6] for background and definitions.

Cannon and Thurston use these two properties to prove that lifts of surface fibers extend continuously to the sphere at infinity of $\widetilde{M}$, and their boundaries therefore give natural examples of Peano-like sphere filling curves.

In [21] and [18], Mosher (following Gabai), generalizes [4] by constructing examples of pseudo-Anosov flows almost transverse to any finite depth co-orientable taut foliation. In [8], Fenley and Mosher prove that these flows are actually quasigeodesic. In particular, quasigeodesic flows exist on any closed hyperbolic 3-manifold which is not a rational homology sphere. 
All known examples of pseudo-Anosov flows are quasigeodesic, and it is consistent with all known examples that all quasigeodesic flows are homotopic (as oriented line fields) to pseudo-Anosov flows. On the other hand, the existence of a pseudo-Anosov flow on a 3-manifold has many important consequences. For example, a pseudo-Anosov flow gives rise to a pair of singular stable and unstable foliations, which can be split open to a pair of transverse genuine laminations. Genuine laminations were introduced in Gabai and Oertel [12], and it is known that they certify many useful properties of a 3-manifold Gabai and Kazez [10;11].

Moreover, in Calegari-Dunfield [3], it is shown that if $M$ admits a pseudo-Anosov flow $X$, then $\pi_{1}(M)$ acts faithfully on a circle by homeomorphisms, and preserves a pair of laminations of this circle. Here a lamination of a circle is the boundary data inherited by the circle at infinity from a geodesic lamination of the hyperbolic plane. Again informally, a lamination of a circle is just a closed, unlinked subset of the space of pairs of distinct points in $S^{1}$. We give more precise definitions in the sequel.

In [3], these (1-dimensional) dynamical properties of fundamental groups of 3manifolds admitting pseudo-Anosov flows were exploited to give the first example of a hyperbolic 3-manifold without a pseudo-Anosov flow. The example is the Weeks manifold, the smallest known closed hyperbolic 3-manifold, which can be obtained for instance by $\left(\frac{5}{1}, \frac{5}{2}\right)$ surgery on the Whitehead link in $S^{3}$.

In this paper, we show that a quasigeodesic flow on a hyperbolic 3-manifold gives rise to a similar dynamical package for $\pi_{1}(M)$ : in particular, we show that for $M$ a hyperbolic 3-manifold with a quasigeodesic flow, there is a faithful action of $\pi_{1}(M)$ on a circle by homeomorphisms, which preserves a pair of laminations of this circle. In this way, our theory lets us promote a codimension 2 structure (a flow on a 3-manifold) to a codimension 1 structure (laminations on a circle). Dually, our theory reduces the analysis of holonomy on a two-dimensional leaf space to the dynamics of $\pi_{1}(M)$ on a compact one-dimensional manifold. Experience has shown that the theory of actions of groups on 1-dimensional objects is rich and profound, whereas the theory of group actions on 2-manifolds remains somewhat elusive; therefore we think that this dimensional reduction is significant.

An important application of our structure theory is that the unit ball of the (dual) Thurston norm on cohomology can be detected by quasigeodesic flows. A basic reference for the Thurston norm is [26]. A flow on a 3-manifold is orthogonal to an oriented 2-plane distribution. Such a distribution has a well-defined integral Euler class, which is an element of $H^{2}(M)$. The Milnor-Wood inequality together with our structure theory implies that the set of classes obtained from quasigeodesic flows are contained in the unit ball of the dual Thurston norm. Conversely, Fenley and Mosher [8; 
18] show that every extremal class is realized by some quasigeodesic pseudo-Anosov flow. In particular, the unit ball can be characterized as the convex hull of such flow classes.

As another important corollary, the calculations of [3] show that the Weeks manifold does not admit a quasigeodesic flow. This is the first known example of a hyperbolic 3 manifold without a quasigeodesic flow, thereby answering in the negative a longstanding question of Thurston. This example is not sporadic: combined with an important recent result of Fenley in [5], our results imply that there are infinitely many closed hyperbolic 3-manifolds without quasigeodesic flows.

\subsection{Orientation convention}

Throughout this paper, we adhere to the convention that all manifolds under consideration are orientable. It follows that the leaf space of all flows are orientable and co-orientable. We remark that it is straightforward to extend the results in this paper to non-orientable 3-manifolds.

\subsection{Statement of results}

In Section 2, we introduce the class of product covered flows. These are flows on a 3-manifold $M$ which lift to give a product structure $\mathbb{R} \times \mathbb{R}^{2}$ on the universal cover $\widetilde{M}$, where the flowlines $l$ are the factors $\mathbb{R} \times$ point. We show that this topological definition is implied by a geometric condition, that flowlines on $\widetilde{M}$ are uniformly properly embedded; that is, that distances in $\widetilde{M}$ as measured along flowlines, and as measured in the hyperbolic metric, are comparable at every scale.

In Section 3 we show that quasigeodesic flows on hyperbolic 3 manifolds are uniformly quasigeodesic. That is, if $X$ is a flow on $M$ such that flowlines on $\widetilde{M}$ are all quasigeodesic, then there is a uniform $k$ such that every flowline is actually $k$-quasigeodesic. In particular, such a flow is uniform in the sense of Section 2, and therefore product covered.

It follows that the natural holonomy representation of $\pi_{1}(M)$ on the leaf space of the flow on $\widetilde{M}$ is actually an action by homeomorphisms on the plane $\mathbb{R}^{2}$.

In Section 4 we introduce natural equivalence relations on this $\mathbb{R}^{2}$, and use the holonomy action of $\pi_{1}(M)$ to construct two universal circles, which parameterize the action at infinity. Our first main result is the following: 
Theorem A Let $M$ be a closed oriented hyperbolic 3-manifold with a quasigeodesic flow $X$. Then there are faithful homomorphisms

$$
\rho_{\text {univ }}^{ \pm}: \pi_{1}(M) \rightarrow \text { Homeo }^{+}\left(\left(S_{\text {univ }}^{1}\right)^{ \pm}\right)
$$

where $\left(S_{\text {univ }}^{1}\right)^{ \pm}$are topological circles, called the universal circles of $X$.

As remarked in the introduction, this easily leads to the corollary that the Weeks manifold admits no quasigeodesic flow.

In Section 5 we compare the two circles $\left(S_{\text {univ }}^{1}\right)^{ \pm}$and show that they can be collated into a single master circle $S_{\text {univ }}^{1}$. More precisely, we prove:

Theorem B Let $M$ be a closed oriented hyperbolic 3-manifold with a quasigeodesic flow $X$. Then there is a canonical circle $S_{\text {univ }}^{1}$, a faithful homomorphism

$$
\rho_{\text {univ }}: \pi_{1}(M) \rightarrow \text { Homeo }^{+}\left(S_{\text {univ }}^{1}\right)
$$

and natural monotone maps

$$
\phi^{ \pm}: S_{\text {univ }}^{1} \rightarrow\left(S_{\text {univ }}^{1}\right)^{ \pm}
$$

so that:

$$
\phi_{*}^{ \pm}\left(\rho_{\text {univ }}\right)=\rho_{\text {univ }}^{ \pm}
$$

Moreover, there are a pair of laminations $\Lambda_{\text {univ }}^{ \pm}$of $S_{\text {univ }}^{1}$ which are preserved by $\pi_{1}(M)$, and satisfy:

$$
\phi^{ \pm}\left(\Lambda_{\text {univ }}^{ \pm}\right)=\Lambda^{ \pm}
$$

Finally, in Section 6 we deduce some corollaries of our structure theory. We prove:

Theorem C Let $M$ be a closed oriented hyperbolic 3-manifold. Then the convex hull of the set of Euler classes $e(X)$ as $X$ varies over the set of quasigeodesic flows on $M$ is exactly the unit ball of the dual Thurston norm on $H^{2}(M)$.

Note that one direction of this theorem - that the convex hull contains the unit ball is due to Gabai, Mosher [18] and Fenley-Mosher [8].

This theorem generalizes an earlier theorem of Mosher (see [19] and [20]), who showed that the Euler class of a pseudo-Anosov quasigeodesic flow is contained in the unit ball of the dual Thurston norm. Mosher argues that a pseudo-Anosov quasigeodesic flow can be isotoped to meet any norm-minimizing surface $S$ in a hyperbolic 3-manifold "efficiently"; the relevant inequality follows easily from this. Our methods give a new and logically independent proof of Mosher's theorem, but do not give a new proof of the existence of such an efficient isotopy class of a flow. 


\subsection{Acknowledgements}

I would like to thank Nathan Dunfield for a number of valuable comments and corrections. I would especially like to thank Lee Mosher for bringing my attention to some important references, and for making many detailed and constructive comments and corrections. While this research was carried out, I was partially supported by the Sloan foundation.

\section{Product covered flows}

A flow on a manifold $M$ is just a $C^{1}$ action of $\mathbb{R}$. That is, a $C^{1}$ map

$$
X: \mathbb{R} \times M \rightarrow M
$$

such that $X(t, X(s, m))=X(t+s, m)$ for all $t, s \in \mathbb{R}$ and $m \in M$. A flow is nonsingular if this action is locally free; ie, for each $p \in M$ and each $t \in \mathbb{R}$, the derivative

$$
\frac{d}{d t} X(p, t)
$$

is nonvanishing. In this case, the orbits of $X$ give an oriented 1-dimensional foliation of $M$, which we denote $X_{\mathfrak{r}}$. Two different nonsingular flows define the same foliation if and only if they differ by a reparameterization; conversely, a nonsingular 1-dimensional oriented foliation defines a flow by parameterizing each flowline by arclength.

Definition 2.1 Let $\widetilde{X_{\mathfrak{\gamma}}}$ be the pulled back foliation of a flow $X$ on $M$. The leaf space of $\widetilde{X}_{\mathscr{Y}}$ is the quotient space of $\widetilde{M}$ by the equivalence relation $p \sim q$ if $p$ and $q$ are contained in the same flowline of $\widetilde{X}_{\mathfrak{\wp}}$.

The leaf space of $\widetilde{X_{\widetilde{\gamma}}}$ is rarely Hausdorff, but if it is, it is a simply connected 2-manifold. The interesting case for our analysis will be where this leaf space is $\mathbb{R}^{2}$ rather than $S^{2}$

Definition 2.2 A flow $X$ on a 3-manifold $M$ is called product covered if the pullback of the foliation $X_{\widetilde{S}}$ to the universal cover $\widetilde{M}$ is topologically equivalent to the product foliation of $\mathbb{R}^{3}$ by vertical lines.

If $X$ is product covered, we denote the quotient map from $\widetilde{M}$ to the leaf space of $\widetilde{X_{\Im}}$ by

$$
\pi_{X}: \widetilde{M} \rightarrow \mathbb{R}^{2}
$$

Example 2.3 A linear flow on the torus $T^{3}$ lifts to a linear flow on $\mathbb{R}^{3}$, and is therefore product covered. 
Example 2.4 Let $M$ be a 3-manifold, and let (3) be an $\mathbb{R}$-covered foliation. That is, a co-oriented codimension one foliation such that the space of leaves of the pullback foliation $\widetilde{\mathbb{G}}$ of the universal cover $\widetilde{M}$ is homeomorphic to $\mathbb{R}$. A transverse flow $X$ is regulating if every leaf of $\widetilde{\mathfrak{S}}$ intersects every flowline of $\widetilde{X_{\mathfrak{\mho}}}$. Every regulating flow is product covered. Moreover, every $\mathbb{R}$-covered foliation admits a regulating flow. See [1] or [7] for details.

Definition 2.5 A flow $X$ is uniform if there is a proper monotone increasing function $f: \mathbb{R}^{+} \rightarrow \mathbb{R}^{+}$such that for any flowline $l$ of $\widetilde{X}_{\mathfrak{\mho}}$, and any two points $p, q \in l$, there is an inequality

$$
d_{\widetilde{M}}(p, q) \geq f\left(d_{l}(p, q)\right)
$$

with respect to any fixed Riemannian metric on $\widetilde{M}$ pulled back from $M$.

In other words, there is a uniform comparison between distance as measured in a flowline, and distance as measured in $\widetilde{M}$. Notice that with this definition, if $M$ is a circle bundle over $S^{2}$, and $X$ is the flow which rotates the circles at some speed, then $X$ is uniform. On the other hand, this case is exceptional: we show now that if $M$ is not a circle bundle over $S^{2}$, any uniform flow is product covered.

Lemma 2.6 Suppose $X$ is a flow on a closed 3-manifold $M$. If $X$ is uniform and $M$ is not a circle bundle over a sphere, then $X$ is product covered.

Proof Suppose the leaf space of $\widetilde{X_{\breve{\gamma}}}$ is not Hausdorff. Then there are distinct flowlines $l, m$ of $\widetilde{X}_{\mathscr{\mho}}$ containing points $p \in l, q \in m$, and a sequence of flowlines $l_{i}$ of $\widetilde{X}_{\mathscr{F}}$ and points $p_{i}, q_{i} \in l_{i}$ such that $p_{i} \rightarrow p, q_{i} \rightarrow q$. Since the sequences $p_{i}$ and $q_{i}$ are convergent, the distance $d_{\widetilde{M}}\left(p_{i}, q_{i}\right)$ is eventually bounded by $d_{\widetilde{M}}(p, q)+\epsilon$. On the other hand, since flowlines converge on compact subsets, the distance between $p_{i}$ and $q_{i}$ in $l_{i}$ goes to infinity. But this violates uniformity. It follows that for $X$ uniform, the leaf space of $\widetilde{X_{\mathfrak{Y}}}$ is Hausdorff.

If the leaf space $\widetilde{X}_{\mathfrak{\mho}}$ is Hausdorff, it is an open, simply connected 2-manifold; in particular, it is either a plane, in which case $X$ is product covered, or a sphere, in which case $\widetilde{M}$ fibers over $S^{2}$, and $M$ is a circle bundle over $S^{2}$, as required.

\section{Quasigeodesic flows are uniformly quasigeodesic}

In this section we show that a flow on a hyperbolic 3-manifold, all of whose flowlines lift in the universal cover to quasigeodesics, is actually uniformly quasigeodesic. In particular, such a flow is uniform, and therefore product covered. This discussion involves the basic elements of the theory of coarse geometry; a reference is [13]. 
Definition 3.1 Let $X, d_{X}$ and $Y, d_{Y}$ be metric spaces. A map $\phi: X \rightarrow Y$ is a $k$ quasi-isometric embedding if there is a constant $k \geq 1$ such that for all $p, q \in X$,

$$
\frac{1}{k} d_{Y}(\phi(p), \phi(q))-k \leq d_{X}(p, q) \leq k d_{Y}(\phi(p), \phi(q))+k
$$

If $X, d_{X}$ is isometric to $\mathbb{R}$, the image of $X$ under a $k$ quasi-isometric embedding is a $k$ quasigeodesic.

If $k$ is understood or undetermined, we talk about quasi-isometric embeddings and quasigeodesics respectively.

Remark 3.2 One also says that $\phi: X \rightarrow Y$ is a $(k, \epsilon)$ quasi-isometric embedding if there are constants $k \geq 1, \epsilon \geq 0$ such that for all $p, q \in X$,

$$
\frac{1}{k} d_{Y}(\phi(p), \phi(q))-\epsilon \leq d_{X}(p, q) \leq k d_{Y}(\phi(p), \phi(q))+\epsilon
$$

The two notions are obviously equivalent, at the cost of possibly increasing the constants. For the sake of notational simplicity, we prefer to work with the first definition.

The following elementary lemma is well-known. A proof is found in [15].

Lemma 3.3 Let $\gamma$ be a $k$ quasigeodesic segment, ray or line in $\mathbb{Q}^{n}$. Then there is a constant $C(k)$ depending only on $k$ and a geodesic $\gamma_{g}$ with the same (possibly ideal) endpoints, such that $\gamma$ and $\gamma_{g}$ are distance at most $C(k)$ apart in the Hausdorff metric.

Remark 3.4 The constant $C(k)=2^{8} k^{4}$ suffices in Lemma 3.3.

Definition 3.5 A $C^{1}$ flow $X$ on a 3 -manifold is quasigeodesic if each leaf $l$ of $\widetilde{X_{\mathfrak{Y}}}$ with its induced length metric is a quasigeodesic in $\widetilde{M}$. A flow $X$ is uniformly quasigeodesic if there is a constant $k$ such that each leaf $l$ of $\widetilde{X_{\mathfrak{F}}}$ is a $k$ quasigeodesic.

Remark 3.6 Note that since $M$ is compact and $X$ is $C^{1}$, the parameterizations of leaves $l$ of $\widetilde{X_{\mathfrak{Y}}}$ by arclength or by the flow are uniformly comparable.

Remark 3.7 The $C^{1}$ condition is superfluous here. All we really need is that flowlines are rectifiable, and distance along segments in flowlines varies continuously in the Hausdorff topology.

The following definition is due to Gromov: 
Definition 3.8 (Gromov) A path $l$ in $\mathbb{\boxplus}^{n}$ is locally $k$ quasigeodesic on the scale $c$ if for all pairs of points $p, q \in l$ which are distance $t \leq c$ apart in $l$, the points $p, q$ are distance $\geq t / k-k$ apart in $\mathbb{\boxplus}^{n}$. If the number $c$ is understood, we just say $l$ is locally $k$ quasigeodesic.

The following lemma is the content of $\left[13\right.$, Remark 7.2.B], applied to paths in $\mathbb{Q}^{n}$ :

Lemma 3.9 (Gromov) For every $k \geq 1$ there is a universal scale $c(k)$ such that if $l$ is a path in $\mathbb{H}^{n}$ which is locally $k$ quasigeodesic on the scale $c(k)$, then $l$ is (globally) $2 k$ quasigeodesic.

In particular, if $l$ is a path in $\mathbb{\boxplus}^{n}$ which is not $2 k$ quasigeodesic, there is a subsegment of $l$ of length $\leq c(k)$ which is not $k$ quasigeodesic. The function $c(k)=1000 k^{2}$ works for sufficiently large $k$.

Lemma 3.10 Let $M$ be a closed hyperbolic 3-manifold. Then every quasigeodesic flow $X$ on $M$ is uniformly quasigeodesic.

Proof Suppose to the contrary that we can find a sequence $l_{i}$ of flowlines of $\widetilde{X}_{\mathscr{\mho}}$ which are $k_{i}$ quasigeodesic for some minimal $k_{i}$, where $k_{i} \rightarrow \infty$. By refining the sequence if necessary, we can assume $k_{i}>2^{i}$. So $l_{i}$ is not $2^{i}$ quasigeodesic, and therefore by Lemma 3.9, it contains a segment $l_{i}^{i}$ of length at most $c\left(2^{i}\right)$ which is not $2^{i-1}$ quasigeodesic. But then $l_{i}^{i}$ contains a subsegment $l_{i}^{i-1}$ of length at most $c\left(2^{i-1}\right)$ which is not $2^{i-2}$ quasigeodesic, and so on. Continuing inductively, we find a nested sequence of segments

$$
l_{i}^{n} \subset l_{i}^{n+1} \subset \cdots \subset l_{i}^{i}
$$

where each $l_{i}^{j}$ has length at most $c\left(2^{j}\right)$, and is not $2^{j-1}$ quasigeodesic. Here $n$ is some universal constant (eg $n=100$ ) which should be fixed independently of $i$. Let $p_{i}$ be the midpoint of $l_{i}^{n}$, and fix a sequence of elements $\alpha_{i} \in \pi_{1}(M)$ such that $\alpha_{i}\left(p_{i}\right)$ are contained in a fixed fundamental domain of $\widetilde{M}$. Choose a convergent subsequence, so that $\alpha_{i}\left(p_{i}\right) \rightarrow p$ where $p$ is contained in some flowline $l$. Since the flowlines $\alpha_{i}\left(l_{i}\right)$ converge on compact subsets, it follows that the nested segments

$$
\alpha_{i}\left(l_{i}^{n}\right) \subset \alpha_{i}\left(l_{i}^{n+1}\right) \subset \cdots \subset \alpha_{i}\left(l_{i}^{i}\right) \subset \alpha_{i}\left(l_{i}\right)
$$

all converge termwise to

$$
l^{n} \subset l^{n+1} \subset l^{n+2} \subset \cdots \subset l
$$

To see this, observe that each $\alpha_{i}\left(l_{i}^{j}\right)$ has length bounded by $c\left(2^{j}\right)$, and contains a point $\alpha_{i}\left(p_{i}\right)$ in a fixed fundamental domain. So the family $\alpha_{i}\left(l_{i}^{j}\right)$ is precompact, and 
some subsequence converges to some $l^{j}$. Since $l^{j}$ is contained in $l$, it is unique, and therefore we did not actually need to pass to a subsequence to get convergence.

By construction, for each $k$, each $l_{j}^{k}$ is not $2^{k-1}$ quasigeodesic, so the same is true of $l^{k}$. It follows that $l$ is not $2^{k}$ quasigeodesic for any $k$. But $l$ is a flowline of $X$, so this violates the hypothesis.

This contradiction proves the lemma.

Remark 3.11 Gromov's lemma holds for an arbitrary $\delta$-hyperbolic geodesic metric space, where now we must insist that our paths are locally $k$ quasigeodesic on the scale $c(k, \delta)$. It follows that Lemma 3.10 also applies to $\delta$-hyperbolic 3-manifolds.

Theorem 3.12 Let $X$ be a quasigeodesic flow on a closed hyperbolic 3-manifold. Then $X$ is product covered.

Proof By Lemma 3.10, the flowlines of $\widetilde{X_{\mathfrak{\mho}}}$ are uniformly $k$ quasigeodesic for some $k$. In particular, the flow $X$ is uniform. But then the theorem follows immediately from Lemma 2.6

Notation 3.13 If $X$ is a quasigeodesic flow on a closed hyperbolic 3-manifold $M$, we denote the leaf space of $\widetilde{X}_{\mathfrak{Y}}$ by $P_{X}$. Note by Theorem 3.12 that $P_{X}$ is homeomorphic to $\mathbb{R}^{2}$.

Example 3.14 The following example is found in [29]. Let $M$ be a surface bundle over a circle, and let (S) be the foliation by surfaces. Let $X$ be any transverse flow. Then flowlines of $\widetilde{X_{\breve{\mho}}}$ are uniformly quasigeodesic. To see this, let $\alpha$ be a nonsingular closed 1 -form whose kernel is tangent to (5). Then $\alpha(X)$ is bounded below by some $\epsilon>0$, whereas $|\alpha|$ (with respect to the hyperbolic metric) is bounded above by some $K$. It follows that flowlines of $\widetilde{X_{\breve{F}}}$ are uniformly $K / \epsilon$ quasigeodesic.

Example 3.15 This generalizes the previous example. A closed 3-manifold $M$ is said to slither over $S^{1}$ if the universal cover of $M$ fibers over $S^{1}$

$$
s: \widetilde{M} \rightarrow S^{1}
$$

in such a way that $\pi_{1}(M)$ acts by bundle automorphisms. Note that the fibers of $s$ are disconnected. Suppose $M$ slithers over $S^{1}$, and suppose $X$ is a flow on $M$ such that for each flowline $l$ of $\widetilde{X_{\mathfrak{Y}}}$ the map $s: l \rightarrow S^{1}$ is a (universal) covering map. Then by compactness of $M$, there is a universal constant $C$ such that every segment $\sigma$ of a flowline $l$ of $\widetilde{X_{\mathfrak{\zeta}}}$ of length at least $C$ maps surjectively to $S^{1}$ under $s$. On the other 
hand, also by compactness, there is an $\epsilon$ such that every arc in $\widetilde{M}$ of length $\leq \epsilon$ does not map surjectively to $S^{1}$ under $s$. It follows that flowlines of $\widetilde{X_{\overparen{\mho}}}$ are uniformly $C / \epsilon$ quasigeodesic.

Example 3.16 In [21], Mosher constructs an example of a hyperbolic 3-manifold $M$ containing a quasifuchsian surface $S$, and a flow $X$ on $M$ such that away from a single closed orbit $\gamma$, every flowline intersects $S$. It follows that every flowline of $\widetilde{X_{\mathfrak{V}}}$ either crosses lifts of $S$ with definite frequency, or else spends a lot of time very close to lifts of $\gamma$. In either case the flowline is quasigeodesic, and therefore flowlines are uniformly quasigeodesic.

\section{Construction of the universal circles}

In this section we construct two universal circles for a quasigeodesic flow $X$ on a hyperbolic 3-manifold, and show that there are two natural faithful homomorphisms from $\pi_{1}(M)$ to $\mathrm{Homeo}^{+}\left(S^{1}\right)$. Throughout this section and the following one, we make use of some elementary properties of circular orders and the order topology.

\subsection{Circular orders and the order topology}

We collect here relevant definitions and basic facts about circular orders.

Definition 4.1 Let $S$ be a set. A circular order on $S$ is a cochain

$$
\gamma: S \times S \times S \rightarrow\{-1,0,1\}
$$

which takes the value 0 if and only if two or more of its co-ordinates are equal, and which satisfies a cocycle condition:

$$
\gamma(a, b, c)-\gamma(a, b, d)+\gamma(a, c, d)-\gamma(b, c, d)=0
$$

for all quadruples $a, b, c, d \in S$.

At least for sets $S$ of sufficiently small cardinality, one can think of a circular order on $S$ as an embedding of $S$ in an oriented $S^{1}$ in such a way that a triple $(a, b, c)$ takes the value $-1,0,1$ if and only if the three points occur in clockwise, degenerate or anticlockwise order in $S^{1}$, with respect to the orientation on $S^{1}$. We now make this intuition precise.

A circular order on $S$ determines a topology, called the order topology. A basis for this topology consists of sets $U_{a, b}$ with $a, b \in S$ defined by

$$
U_{a, b}=\{c \in S \mid \gamma(a, b, c)=-1\}
$$


which we call open intervals, and whose complements $S \backslash U_{a, b}$ are called closed intervals.

A circularly ordered set is order complete if any nested infinite sequence of closed intervals has a non-empty intersection.

Any circularly ordered set $S$ has a canonical order completion $\bar{S}$ in which $S$ is dense. If $\bar{S}$ is separable, it is compact, and admits a proper continuous embedding $e: \bar{S} \rightarrow S^{1}$ whose image is closed, and which is unique up to post-composition with an orientationpreserving homeomorphism of $S^{1}$. Any group $G$ of automorphisms of $S$ which preserves the circular order extends to a group of automorphisms of the order completion $\bar{S}$. Moreover, since the embedding of $\bar{S}$ in $S^{1}$ is unique up to homeomorphism, the action of $G$ on $e(\bar{S})$ extends to an action on $S^{1}$ by homeomorphisms.

A reference for this material is [2] or [25].

\subsection{Endpoint maps}

We first construct natural endpoint maps from the leaf space of $\widetilde{X_{\mathscr{\mho}}}$ to the Gromov boundary $S_{\infty}^{2}$ of $\widetilde{M}=\mathbb{M}^{3}$.

Construction 4.2 Let $X$ be a quasigeodesic flow on a closed hyperbolic 3-manifold $M$. By Lemma 3.10, the flowlines of $\widetilde{X}_{\mathfrak{\mho}}$ are uniformly $k$ quasigeodesic. By Theorem 3.12, the leaf space $P_{X}$ of $\widetilde{X_{\mathfrak{F}}}$ is homeomorphic to $\mathbb{R}^{2}$. In particular, the action of $\pi_{1}(M)$ on $\widetilde{M}$ induces a holonomy representation

$$
\rho_{\mathrm{hol}}: \pi_{1}(M) \rightarrow \text { Homeo }^{+}\left(P_{X}\right)
$$

Since each flowline $l$ of $\widetilde{X_{\overparen{r}}}$ is a quasigeodesic, there are two well-defined endpoint maps

$$
e^{ \pm}: P_{X} \rightarrow S_{\infty}^{2}
$$

where $S_{\infty}^{2}$ denotes the sphere at infinity of $\mathbb{\boxplus}^{3}=\widetilde{M}$. Thinking of a flowline $l$ of $\widetilde{X_{\mathfrak{r}}}$ as a point in the leaf space $P_{X}$, we define $e^{ \pm}(l)$ to be the positive and negative endpoints of the unique oriented geodesic in $\mathbb{}^{3}$ which is a finite Hausdorff distance from $l$.

Lemma 4.3 The endpoint maps $e^{ \pm}$are continuous.

Proof Suppose $l$ is a complete $k$ quasigeodesic in $\mathbb{\boxplus}^{3}$. Then by Lemma 3.3, the geodesic $l_{g}$ with the same endpoints is contained in the $2^{8} k^{4}$ neighborhood of $l$, and vice versa. In particular, if $l_{i}$ is a sequence of flowlines of $\widetilde{X}_{\mathfrak{\gamma}}$ which converges on 
compact subsets to $l$, then the straightened geodesics $\left(l_{i}\right)_{g}$ eventually contain arbitrarily long segments which are contained in the $2^{9} k^{4}$ neigbhorhood of $l_{g}$. If $\left(l_{i}\right)_{g}, l_{g}$ are two hyperbolic geodesics which are $2^{9} k^{4}$ close on a segment of length $t$, then they are $2^{9} k^{4} e^{-t}$ close on a subsegment of length $t / 2$. It follows that the straightened geodesics $\left(l_{i}\right)_{g}$ converge to $l_{g}$ on compact sets, and therefore the endpoint maps are continuous.

Lemma 4.4 The images $e^{ \pm}\left(P_{X}\right)$ are both dense in $S_{\infty}^{2}$.

Proof Since $M$ is a closed hyperbolic 3-manifold, any non-empty $\pi_{1}(M)$-invariant subset of $S_{\infty}^{2}$ is dense. The lemma follows.

Lemma 4.5 Let $\gamma \subset P_{X}$ be an embedded circle, and $D \subset P_{X}$ the region bounded by $\gamma$. Then there is an equality of images

$$
e^{ \pm}(\gamma)=e^{ \pm}(D)
$$

Proof For concreteness we concentrate on $e^{+}$. Suppose to the contrary that there is $p \in e^{+}(D)$ which is not in the image of $e^{+}(\gamma)$.

Let $\sigma: D \rightarrow \widetilde{M}$ be a section of $\pi_{X}$. That is, we suppose that

$$
\pi_{X} \circ \sigma: D \rightarrow D
$$

is the identity.

Let $S \subset \widetilde{M}$ be the union of $\sigma(D)$ and the positive rays contained in the flowlines of $\widetilde{X_{\mathfrak{F}}}$ which emanate from $\sigma(\gamma)$. Then the positive rays contained in flowlines of $\widetilde{X}_{\mathscr{\gamma}}$ which emanate from $\sigma(D)$ limit to points in $e^{+}(D)$. Orient $S$ so that the positive side of $S$ is the side which contains these positive rays. Since flowlines of $\widetilde{X}_{\mathfrak{Y}}$ are uniformly quasigeodesic, the closure of $S$ in $\widetilde{M}$ is just $S \cup e^{+}(\gamma)$. Then for any point $q \in e^{+}(D)$ which is not in $e^{+}(\gamma)$, and for any sequence $q_{i} \in \mathbb{M}^{3}$ limiting to $q \in S_{\infty}^{2}$, the sequence $q_{i}$ is eventually contained on the positive side of $S$.

Now, by Lemma 4.4 , there is some flowline $l$ of $\widetilde{X}_{\mathscr{Y}}$ with $e^{-}(l)$ arbitrarily close to $p$. It follows that the negative end of $l$ is contained on the positive side of $S$. But if any point $r \in l$ is on the positive side of $S$, then the negative ray contained in $l$ must intersect $\sigma(D)$, and the negative end of $l$ is contained on the negative side of $S$. This contradiction proves the lemma. 


\subsection{Separation properties}

We will study the separation properties in $P_{X}$ of the various point preimages of $e^{ \pm}$. The basic algebraic tool we use is Alexander duality. Since these are arbitrary closed sets, singular cohomology is insufficient for our purposes.

We recall the definition of the Alexander cohomology theory, following [23, Section 6.4]. For a topological space $X$ and a coefficient module $G$, define $C^{q}(X ; G)$ to be the module of all functions $\varphi$ from $X^{q+1}$ to $G$ with addition and scalar multiplication defined pointwise. An element $\varphi \in C^{q}(X)$ is said to be locally zero if there is a covering of $X$ by open sets such that $\varphi$ vanishes on any $(q+1)$-tuple which lies in some element of the covering. Define $\bar{C}^{*}(X)$ to be the quotient complex of $C^{q}(X ; G)$ by the locally zero cochains, and denote the resulting cohomology theory by $\bar{H}^{*}(X ; G)$.

Alexander cohomology has the following property, which the reader can take as a working definition:

Theorem 4.6 [23, Corollary 6.9.9] If $A$ is any closed subset of a manifold $X$, then as $U$ varies over neighborhoods of $A$ in $X$,

$$
\lim _{\rightarrow}\left\{H^{*}(U ; G)\right\} \cong \bar{H}^{*}(A ; G)
$$

Moreover, we have the following characterization of connectedness:

Theorem 4.7 [23, Corollary 6.5.7] A nonempty space $X$ is connected if and only if

$$
G \cong \bar{H}^{0}(X ; G)
$$

We can now deduce a fundamental property of the point preimages under the maps $e^{ \pm}$.

Lemma 4.8 For every point $p \in S_{\infty}^{2}$ in the image of $e^{+}$, every connected component of $\left(e^{+}\right)^{-1}(p)$ is unbounded, and similarly for $e^{-}$.

Proof We suppose not and arrive at a contradiction. For ease of notation, define $L=\left(e^{+}\right)^{-1}(p)$. Then $L$ is closed. Let $K$ be a bounded component of $L$. Then $K$ is compact.

If $L$ is compact, then $L$ can be included into the interior of a compact disk in $P_{X}$ whose boundary separates $L$ from infinity, contrary to Lemma 4.5.

Otherwise, $L$ is unbounded. We take $\widehat{L}$ to be the closure of $L$ in $S^{2}=P_{X} \cup \infty$; ie $\widehat{L}=L \cup \infty$. Notice that since $K$ is compact, it is not contained in the connected component which contains the point $\infty$, and therefore $\hat{L}$ is not connected. 
By Theorem 4.7, we have $\bar{H}^{0}(\widehat{L} ; \mathbb{R}) \neq \mathbb{R}$. Moreover, by Theorem 4.6, there is an open set $U \subset S^{2}$ with $H^{0}(U ; \mathbb{R}) \neq \mathbb{R}$, each component of which intersects $\widehat{L}$. It follows that $U$ has at least two components, and therefore one component $U_{1}$ is separated from $U_{2}:=U \backslash U_{1}$ with $\infty \in U_{2}$. Let $K_{1}$ be a component of $\widehat{L}$ which is contained in $U_{1}$, and let $\gamma$ be a loop in $S^{2} \backslash U$ which separates $U_{1}$ from $U_{2}$. Then $\gamma$ separates $K_{1}$ from infinity, and bounds a disk $D \subset P_{X}$ which contains $K_{1}$ in its interior. On the other hand, $e^{+}(\gamma)$ does not contain $p$. This contradicts Lemma 4.5 and proves the lemma.

Corollary 4.9 For every point $p \in S_{\infty}^{2}$ in the image of $e^{+}$and every connected component $K$ of $\left(e^{+}\right)^{-1}(p)$, the connected components of $P_{X} \backslash K$ are unbounded disks.

Proof Since $K$ is connected, closed and unbounded, complementary regions are simply-connected, by Theorem 4.6 and standard Alexander duality for polyhedra in manifolds. If $S$ is a bounded complementary region, and $p \in S$ is arbitrary, then set $q=e^{+}(p)$, and let $L$ be the connected component of $\left(e^{+}\right)^{-1}(q)$ containing $p$. Since $L$ and $K$ are closed and disjoint, they have disjoint open neighborhoods. It follows that $L$ is contained in some closed subset of $S$. In particular, this implies that $L$ is bounded, contrary to Lemma 4.8 .

Construction 4.10 Let $e^{ \pm}: P_{X} \rightarrow S_{\infty}^{2}$ be the endpoint maps from Construction 4.2. These maps define equivalence relations $\sim^{ \pm}$on $P_{X}$, where the equivalence classes of $\sim^{+}$are the connected components of the preimages of points in $S_{\infty}^{2}$ under $e^{+}$. The relation $\sim^{-}$is defined similarly.

Since the maps $e^{ \pm}$are natural, the equivalence classes of $\sim^{ \pm}$are permuted by $\pi_{1}(M)$ and the maps $e^{ \pm}$factor through the quotient spaces $T^{ \pm}=P_{X} / \sim^{ \pm}$. Let

$\pi^{ \pm}: P_{X} \rightarrow T^{ \pm}$
denote the quotient maps, and $\quad \iota^{ \pm}: T^{ \pm} \rightarrow S_{\infty}^{2}$

the induced maps on the factor spaces, so that $\iota^{ \pm} \circ \pi^{ \pm}=e^{ \pm}$.

\subsection{Ends and circular orders}

Given a space $X$, the ends of $X$ (denoted $\mathfrak{S}(X))$ may be defined informally as the connected components of the complement of arbitrarily big compact subsets. More precisely, if $K_{i}$ is an exhaustion of $X$ by compact subsets, and $U_{i j}$ are the connected components of $X \backslash K_{i}$, each $U_{i j}$ includes into a unique $U_{i^{\prime} j^{\prime}}$ for all $i^{\prime}<i$, thereby 
defining a directed system. The inverse limit of this directed system is the set $\mathbb{E}(X)$. If $X$ is a manifold, we may replace the phrase connected component above by path connected component, and think of an end as an equivalence class of properly embedded ray $r$ in $X$, where $r \sim r^{\prime}$ if the unbounded components of $r, r^{\prime} \cap X \backslash C$ are in the same path component of $X \backslash C$, where $C$ is any compact set.

Cohomologically, given a collection of (simplicial, singular, Alexander, etc.) cochains $C^{*}(X)$ with field coefficients (which we supress in our notation), there is a natural subcomplex $C_{c}^{*}(X)$ consisting of cochains with compact support, and a natural quotient complex $C_{e}^{*}(X)$ defined by the short exact sequence

$$
0 \rightarrow C_{c}^{*}(X) \rightarrow C^{*}(X) \rightarrow C_{e}^{*}(X) \rightarrow 0
$$

Then for reasonable spaces (where reasonable depends on the cohomology theory in question), $H_{e}^{0}(X)$ is a vector space with dimension equal to the cardinality of $\mathfrak{S}(X)$.

For Alexander cohomology, we have the following interpretation of cohomology with compact support:

Theorem 4.11 [23, Corollary 6.7.12] If $X$ is a locally compact Hausdorff space and $\hat{X}$ is the one-point compactification of $X$, there is an isomorphism

$$
\bar{H}_{c}^{q}(X ; G) \cong \widetilde{\bar{H}}^{q}(\widehat{X} ; G)
$$

where the tilde denotes reduced Alexander cohomology.

For $K \subset P_{X}$ closed and noncompact, the one point compactification $\widehat{K}$ is just $K \cup \infty \subset$ $S^{2}$. Moreover, Alexander duality for polyhedra generalizes as follows:

Theorem 4.12 [23, Theorem 6.9.10] Let $X$ be an orientable $n$-manifold. For any closed pair $(A, B)$ in $X$ there is an isomorphism

$$
H_{q}(X-B, X-A ; G) \cong \bar{H}_{c}^{n-q}(A, B ; G)
$$

We deduce that

$$
\widetilde{H}_{2-q-1}\left(P_{X} \backslash K ; G\right) \cong \bar{H}_{c}^{q}(K ; G)
$$

for any closed $K \subset P_{X}$. Note for such $K$ that $\bar{H}^{0}(K) \cong \mathbb{R}$ if and only if $K$ is connected, and if $K$ is connected, $\bar{H}_{c}^{0}(K) \cong \mathbb{R}$ if and only if $K$ compact. It follows that for $K$ closed, connected and noncompact, $\mathfrak{S}(K)$ is nonempty.

We may define ends of $K$ in geometric language as follows. A sequence $\left(r_{i}\right)$ of properly embedded rays $r_{i} \subset P_{X}$ limits to an end of $K$ if for every open set $U$ containing $K$, there is a positive integer $N$ such that for all $i, j \geq N$, the rays $r_{i}$ and $r_{j}$ are contained 
in $U$, and moreover for any pair of proper sequences of points $p_{k}$ and $q_{k}$ in $r_{i}$ and $r_{j}$ there is a proper (in $P_{X}$ ) sequence of $\operatorname{arcs} \alpha_{k} \subset U$ joining $p_{k}$ to $q_{k}$. Equivalently, for every simply-connected $U$ containing $K$, the rays $r_{i}$ and $r_{j}$ should be properly homotopic in $U$ for all $i, j \geq N$. Two such sequences limit to the same end, denoted $\left(r_{i}\right) \sim\left(r_{i}^{\prime}\right)$, if the alternating sequence $r_{1}, r_{1}^{\prime}, r_{2}, r_{2}^{\prime}, \ldots$ itself limits to an end of $K$.

Definition 4.13 For each $k \in T^{ \pm}$, thinking of $k$ as a closed, connected subset of $P_{X}$, define $\breve{S}_{k}$ to be the set of ends of $k$. Define

$$
\mathfrak{S}^{+}=\bigcup_{k \in T^{+}} \mathfrak{G}_{k}
$$

and define (5- similarly.

Lemma 4.14 There is a natural circular ordering on the set $\mathfrak{S}^{+}$which is preserved by the action of $\pi_{1}(M)$.

Proof Let $e_{i} \in \mathfrak{C}_{k_{i}}$ be distinct elements of $\mathfrak{S}^{+}$, for $i=1,2,3$. Of course, $k_{i}$ and $k_{j}$ might not be distinct if $e_{i} \neq e_{j}$, but in this case, the ends $e_{i}, e_{j}$ of $k_{i}=k_{j} \subset P_{X}$ are different.

By removing a compact subset from $k_{i}$ in the case $k_{i}=k_{j}$, we can replace the $k_{i}$ by disjoint connected sets $k_{i}^{\prime}$ so that the $e_{i}$ are ends of $k_{i}^{\prime}$. Let $U\left(k_{i}^{\prime}\right)$ be disjoint open neighborhoods of the $k_{i}^{\prime}$ in $P_{X}$. Then we can find disjoint proper rays $r_{i}$ with $r_{i} \subset U\left(k_{i}^{\prime}\right)$ corresponding to the $e_{i}$.

We circularly order the $r_{i}$ as follows. Let $D$ be a sufficiently large closed disk in $P_{X}$ which intersects all the $r_{i}$. Let $p_{i}$ be the unique points on $r_{i} \cap \partial D$ such that $r_{i} \backslash p_{i}$ consists of two components, of which the unbounded one is disjoint from $D$. Then the circular order on $\partial D$ defines a circular order on $p_{i}$ and therefore on $r_{i}$.

To see that this is well-defined, suppose without loss of generality that we replace $r_{1}$ with a different proper ray $r_{1}^{\prime}$ corresponding to the same end of $U\left(e_{1}\right)$. Then there are a sequence of arcs $\alpha_{j}$ from $r_{1}$ to $r_{1}^{\prime}$ which are contained in $U\left(e_{1}\right)$, and which are proper in $P_{X}$. Such arcs are disjoint from the other rays $r_{2}, r_{3}$, and therefore they certify that the circular order of $r_{1}, r_{2}, r_{3}$ agrees with the circular order of $r_{1}^{\prime}, r_{2}, r_{3}$.

Lemma 4.15 Let $e \in \mathfrak{S}^{+}$be arbitrary. Then the stabilizer of $e$ in $\pi_{1}(M)$ is either trivial or cyclic.

Proof If $\alpha \in \pi_{1}(M)$ fixes $e \in \mathfrak{E}_{k}$, then $\rho_{\text {hol }}(\alpha)$ must fix $k$, and therefore $\alpha$ must fix $e^{+}(k) \in S_{\infty}^{2}$. But since $M$ is a closed hyperbolic 3-manifold, the stabilizer of any point in $S_{\infty}^{2}$ is trivial or cyclic, as claimed. 
Construction 4.16 We topologize the circularly ordered sets ${ }^{ \pm}$with the order topology, and take their completions $\overline{\mathscr{C}}^{ \pm}$. This makes them into compact circularly ordered sets, and therefore they are naturally order isomorphic to some uncountable compact subset of $S^{1}$.

By quotienting out complementary intervals to the image of this subset, we get natural surjections to two circles

$$
\overline{\mathrm{C}}^{ \pm} \rightarrow\left(S_{\text {univ }}^{1}\right)^{ \pm}
$$

Since this construction is natural, the action of $\pi_{1}(M)$ on ${ }^{ \pm}$extends to an action on $\overline{\mathrm{S}}^{ \pm}$which factors through to an action on $\left(S_{\text {univ }}^{1}\right)^{ \pm}$.

The induced representations

$$
\rho_{\text {univ }}^{ \pm}: \pi_{1}(M) \rightarrow \operatorname{Homeo}\left(\left(S_{\text {univ }}^{1}\right)^{ \pm}\right)
$$

are actually orientation preserving, since $\rho_{\mathrm{hol}}$ is orientation preserving.

We now prove the first main result of the paper:

Theorem A Let $M$ be a closed oriented hyperbolic 3-manifold with a quasigeodesic flow $X$. Then there are faithful homomorphisms

$$
\rho_{\text {univ }}^{ \pm}: \pi_{1}(M) \rightarrow \text { Homeo }^{+}\left(\left(S_{\text {univ }}^{1}\right)^{ \pm}\right)
$$

where $\left(S_{\text {univ }}^{1}\right)^{ \pm}$are topological circles, called the universal circles of $X$.

Proof In Construction 4.16 we actually construct two natural circles $\left(S_{\text {univ }}^{1}\right)^{ \pm}$and natural homomorphisms

$$
\rho_{\text {univ }}^{ \pm}: \pi_{1}(M) \rightarrow \text { Homeo }^{+}\left(\left(S_{\text {univ }}^{1}\right)^{ \pm}\right)
$$

The maps $\mathfrak{r}^{ \pm} \rightarrow\left(S_{\text {univ }}^{1}\right)^{ \pm}$are 1-1 outside of countably many elements, so we can certainly find points in $\left(S_{\text {univ }}^{1}\right)^{ \pm}$in the image of a unique element of $\mathfrak{r}^{ \pm}$. Call such ends injective. Moreover, since the maps $e^{ \pm}$are nonconstant, the images $e^{ \pm}\left(P_{X}\right)$ are uncountable. It follows that there are points $p \in S_{\infty}^{2}$ in the image of $e^{+}$which are not fixed by any nontrivial $\alpha \in \pi_{1}(M)$, and for which every component $k$ of $\left(e^{+}\right)^{-1}(p)$ has injective ends, and similarly for $e^{-}$. It follows that the natural actions of $\pi_{1}(M)$ on $\left(S_{\text {univ }}^{1}\right)^{ \pm}$are both faithful.

Corollary 4.17 The Weeks manifold does not admit a quasigeodesic flow.

Proof In [3], It is shown that the fundamental group of the Weeks manifold does not act faithfully on a circle. It follows from Theorem A that the Weeks manifold does not admit a quasigeodesic flow. 


\section{Properties of the universal circles}

In this section, by studying the properties of $\sim^{ \pm}$in more detail, we construct a pair of $\pi_{1}(M)$-invariant laminations (to be defined below) $\Lambda^{ \pm}$for $\left(S_{\text {univ }}^{1}\right)^{ \pm}$, and use this structure to produce a single canonical circle $S_{\text {univ }}^{1}$ which maps monotonically to each $\left(S_{\text {univ }}^{1}\right)^{ \pm}$.

\subsection{Constructing laminations}

Lemma 5.1 Some equivalence class $k$ of $\sim^{+}$is separating in $P_{X}$.

Proof We suppose not and derive a contradiction.

If each equivalence class $k$ is nonseparating, then $P_{X} \backslash k$ is connected, and $\widetilde{H}_{0}\left(P_{X} \backslash k\right)$ $=0$. By Theorem 4.12, it follows that $\bar{H}_{c}^{1}(k)=0$ and therefore $\bar{H}^{0}(k) \rightarrow \bar{H}_{e}^{0}(k)$ is surjective. Since $k$ is connected by hypothesis, and noncompact by Lemma 4.8, this implies that $k$ has exactly one end.

Since each equivalence class $k$ of $\sim^{+}$has a single end, we can define a map $r: P_{X} \rightarrow$ $\left(S_{\text {univ }}^{1}\right)^{+}$by sending $k$ to this end. We show first that this map is continuous. Let $k$ be an equivalence class, let $k^{l}, k^{r}$ be two other equivalence classes, and let $e, e^{l}, e^{r}$ denote the three unique ends of these equivalence classes. We can join $k^{l}$ to $k^{r}$ by a compact arc $\tau$ which avoids $k$. Let $I \subset\left(S_{\text {univ }}^{1}\right)^{+}$be the open interval complementary to $e^{l}, e^{r}$ containing $e$.

Suppose $p_{i} \rightarrow p \in k$ is a convergent sequence, and suppose $p_{i}$ is in the equivalence class $k_{i}$. By the definition of the equivalence relation $\sim^{+}$, if $k_{i} \rightarrow K$ in the Hausdorff sense, then $k$ contains a connected component of $K$. Moreover, the connected components of $K$ are contained in equivalence classes of $\sim^{+}$.

If there is some other connected component $k^{\prime}$, then either the unique ends of $k^{\prime}$ and $k$ are on the same side of $k^{l} \cup k^{r} \cup \tau$, or else $k_{i}$ intersects $\tau$ for sufficiently large $i$, and therefore $K \cap \tau$ is nonempty. In the second case, since $\tau$ is arbitrary, it follows that $K$ intersects every arc $\tau$ joining $k^{l}$ to $k^{r}$, and therefore some connected component of $K$ separates $k^{l}$ from $k^{r}$, contrary to our assumption that no equivalence class of $\sim^{+}$ is separating.

It follows that the ends of $k^{\prime}$ and $k$ are on the same side of $k^{l} \cup k^{r} \cup \tau$, and therefore limit to points in $I$. But $I$ was arbitrary, so $k$ and $k^{\prime}$ limit to the same point in $\left(S_{\text {univ }}^{1}\right)^{+}$and therefore the map $r$ is continuous.

We now show that there is a circle $\gamma \subset P_{X}$ such that the map $r: \gamma \rightarrow\left(S_{\text {univ }}^{1}\right)^{+}$has degree 1 . Let $k_{1}, k_{2}, k_{3}$ be three equivalence classes, so that their corresponding ends 
$e_{1}, e_{2}, e_{3}$ are distinct and positively ordered in $\left(S_{\text {univ }}^{1}\right)^{+}$. Moreover, choose the $k_{i}$ such that the $e_{i}$ are the unique ends corresponding to their images in $\left(S_{\text {univ }}^{1}\right)^{+}$. We can do this since all but countably many ends are injective. Pick points $p_{i} \in k_{i}$ and join $p_{i}$ to $p_{i+1}$ by an arc $\alpha_{i}$ in the complement of $k_{i+2}$ (here indices are taken mod $3)$. Such arcs $\alpha_{i}$ exist, since each $k_{i}$ is nonseparating, by hypothesis. Then define $\gamma=\alpha_{1} \cup \alpha_{2} \cup \alpha_{3}$. Since $\alpha_{i}$ is disjoint from $k_{i+2}$, the image $r\left(\alpha_{i}\right)$ avoids the point $r\left(k_{i+2}\right)$. It follows that $r: \gamma \rightarrow\left(S_{\text {univ }}^{1}\right)^{+}$has degree 1 .

But under any continuous map from $P_{X}$ to $S^{1}$, the image of any circle in $P_{X}$ must map to $S^{1}$ by a degree 0 map, since $P_{X}$ is contractible. This contradiction shows that some equivalence class is separating, as claimed.

Remark 5.2 We remark that there is a natural topology on $P_{X} \cup\left(S_{\text {univ }}^{1}\right)^{+}$which gives it a compactification as a closed disk, and similarly for $\left(S_{\text {univ }}^{1}\right)^{-}$.

The topology is easy to define: every bounded open set $U \subset P_{X}$ is still open. If $p \in\left(S_{\text {univ }}^{1}\right)^{+}$is arbitrary, we define a basis for the topology near $p$ as follows. Let $I_{i}$ be a nested sequence of closed intervals in $\left(S_{\text {univ }}^{1}\right)^{+}$which converge to $p$ in the Hausdorff sense, and such that the endpoints $\left(I_{i}\right)^{l},\left(I_{i}\right)^{r}$ correspond to ends $e_{i}^{l}, e_{i}^{r}$ of equivalence classes $k_{i}^{l}, k_{i}^{r}$ of $\sim^{+}$. Let $r_{i}^{l}, r_{i}^{r}$ be proper rays contained in small open tubular neighborhoods of $k_{i}^{l}, k_{i}^{r}$, which are eventually disjoint from every fixed equivalence class $k$ of $\sim^{+}$except for $k_{i}^{l}$ or $k_{i}^{r}$, and which go out the ends corresponding to $e_{i}^{l}, e_{i}^{r}$. Let $\tau_{i}$ be an arc joining the initial point of $r_{i}^{l}$ to the initial point of $r_{i}^{r}$. Then the union $r_{i}^{l} \cup \tau_{i} \cup r_{i}^{r}$ separates $P_{X}$ into two open sets $U, V$, one of which (say $U$ ) contains an end $e_{p^{\prime}}$ of some $k_{p^{\prime}}$ which corresponds to some $p^{\prime} \in \operatorname{int}\left(I_{i}\right)$.

Then we define $U \cup \operatorname{int}\left(I_{i}\right)$ to be an open neighborhood of $p$ in $P_{X} \cup\left(S_{\text {univ }}^{1}\right)^{+}$. With this topology, the union is a Peano continuum; that is, it is nondegenerate, perfectly separable and normal, compact, connected and locally connected. Now, a theorem of Zippin (see [27, Theorem III.5.1]) characterizes the 2-disk in the following way:

Theorem 5.3 (Zippin) Let $C$ be a Peano continuum containing a circle $J$ and satisfying the following three conditions:

(1) $C$ contains an arc that spans $J$ (ie, intersects $J$ only at its endpoints)

(2) Every arc of $C$ that spans $J$ separates $C$

(3) No closed proper subset of an arc spanning $J$ separates $C$.

Then $C$ is homeomorphic to the closed 2-disk with boundary $J$ 
An arc $\bar{l}$ in $P_{X} \cup\left(S_{\text {univ }}^{1}\right)^{+}$which spans $\left(S_{\text {univ }}^{1}\right)^{+}$restricts to a properly embedded line $l$ in $P_{X}$. The relevant separation properties follow readily, and we can conclude that $P_{X} \cup\left(S_{\text {univ }}^{1}\right)^{+}$is homeomorphic to a closed disk. This fact is not used elsewhere in this paper.

Definition 5.4 A lamination $\Lambda$ of $S^{1}$ is a closed subset of the space of unordered distinct pairs of points in $S^{1}$ with the property that for any two elements $\{a, b\}$ and $\{c, d\}$ of $\Lambda$, the pair of points $\{a, b\}$ does not link the pair of points $\{c, d\}$ in $S^{1}$ (though it might share one or both points in common). We also abbreviate this last condition by saying that $\Lambda$ is unlinked as a subset of the space of unordered distinct pairs of points in $S^{1}$. We sometimes refer to elements of $\Lambda$ as leaves.

Remark 5.5 It is straightforward to define linking for pairs of disjoint $S^{0}$ 's in any circularly ordered set.

The existence of a separating equivalence class lets us define naturally a pair of laminations of $\left(S_{\text {univ }}^{1}\right)^{ \pm}$.

Construction 5.6 Let $k$ be an equivalence class of $\sim^{+}$. If $k$ is separating, then $k$ has at least two ends, as in the proof of Lemma 5.1. Let $\mathfrak{G}_{k}$ denote the set of ends of $k$, and let $\overline{\mathfrak{5}_{k}}$ denote its closure in $\overline{\mathfrak{S}}^{+}$.

We let $\Lambda_{k}$ be equal to the set of pairs of endpoints of closures of complementary intervals of $\overline{\mathfrak{E}_{k}}$. Note that if $k \neq k^{\prime}$ then $\mathfrak{E}_{k}$ and $\mathfrak{S}_{k^{\prime}}$ do not link. For, $k, k^{\prime}$ are disjoint in $P_{X}$, so any two ends of $k^{\prime}$ are contained on the same side of $k$, and vice versa. Since the stabilizer of $k$ in $\pi_{1}(M)$ is at most cyclic, if we let $k$ be some separating equivalence class of $\sim^{+}$, and $k^{\prime}=\alpha(k)$ for some $\alpha$ not in the stabilizer of $k$, we see that $\mathfrak{E}_{k}$ is not dense in $\mathfrak{S}^{+}$.

Conversely, if $U$ is a connected component of $P_{X} \backslash k$, then we can find $k^{\prime} \subset U$ such that the image of $\mathfrak{S}_{k^{\prime}}$ is disjoint from $\mathfrak{S}_{k}$ in $\left(S_{\text {univ }}^{1}\right)^{+}$. Since we can do this for each component $U$, we see that distinct points of $\overline{\mathfrak{E}_{k}}$ cannot become identified under the quotient map $\overline{\mathrm{S}}^{+} \rightarrow\left(S_{\text {univ }}^{1}\right)^{+}$. It follows that $\Lambda_{k}$ is nonempty as a lamination of $\left(S_{\text {univ }}^{1}\right)^{+}$.

Now, define

$$
\Lambda^{+}=\overline{\bigcup_{k} \Lambda_{k}}
$$

where $k$ ranges over equivalence classes of $\sim^{+}$.

We summarize this construction in a lemma: 
Lemma 5.7 The representations $\rho_{\text {univ }}^{ \pm}$preserve nonempty laminations $\Lambda^{ \pm}$of $\left(S_{\text {univ }}^{1}\right)^{ \pm}$.

Proof This follows from Lemma 5.1 and Construction 5.6.

Lemma 5.8 Let $k^{+}, k^{-} \subset P_{X}$ be equivalence classes of $\sim^{+}, \sim^{-}$respectively. Then the intersection $k^{+} \cap k^{-}$is compact.

Proof By definition, if $K=k^{+} \cap k^{-}$is the intersection, then every two flowlines $l_{1}, l_{2}$ of $\widetilde{X}_{\mathscr{\mho}}$ corresponding to points $l_{1}, l_{2} \in K$ have the same endpoints in $S_{\infty}^{2}$. By Lemma 3.10 and Lemma 3.3, the flowlines $l_{1}$ and $l_{2}$ are distance $\leq 2^{9} k^{4}$ apart in the Hausdorff metric. In particular, the union $\widetilde{X}_{\mathfrak{⿰}}(K)$ of all flowlines corresponding to points in $K$ is itself a finite Hausdorff distance from a geodesic, and therefore intersects a cross-section of the flow in a compact set.

Lemma 5.8 lets us directly compare the circles $\left(S_{\text {univ }}^{1}\right)^{+}$and $\left(S_{\text {univ }}^{1}\right)^{-}$.

Theorem B Let $M$ be a closed oriented hyperbolic 3-manifold with a quasigeodesic flow $X$. Then there is a canonical circle $S_{\text {univ }}^{1}$, a faithful homomorphism

$$
\rho_{\text {univ }}: \pi_{1}(M) \rightarrow \text { Homeo }^{+}\left(S_{\text {univ }}^{1}\right)
$$

and natural monotone maps

$$
\phi^{ \pm}: S_{\text {univ }}^{1} \rightarrow\left(S_{\text {univ }}^{1}\right)^{ \pm}
$$

so that

$$
\phi_{*}^{ \pm}\left(\rho_{\text {univ }}\right)=\rho_{\text {univ }}^{ \pm}
$$

Moreover, there are a pair of laminations $\Lambda_{\text {univ }}^{ \pm}$of $S_{\text {univ }}^{1}$ which are preserved by $\pi_{1}(M)$, and satisfy

$$
\phi^{ \pm}\left(\Lambda_{\text {univ }}^{ \pm}\right)=\Lambda^{ \pm}
$$

Proof We define a circular order on $\mathfrak{b}^{-}=\mathfrak{b}^{+} \cup \mathfrak{S}^{-}$as follows. If $e_{1}, e_{2}, e_{3}$ are ends of $k_{1}, k_{2}, k_{3}$, each of which is an equivalence class of either $\sim^{+}$or $\sim^{-}$, then by Lemma 5.8, outside some compact ball $D$, the subsets $k_{i}$ are disjoint, and we can take disjoint open neighborhoods around them and find proper rays $r_{i}$ contained in these neighborhoods which go out the ends corresponding to $e_{i}$. Since the rays $r_{i}$ are disjoint, they admit a natural circular order, which defines a circular order on $\mathfrak{E}$, which restricts to the circular order on each of $5^{+}$, (5- obtained in Lemma 4.14.

As in Construction 4.16, we can take the order completion $\overline{\mathfrak{S}}$ of $\mathbb{5}$, embed it in an order-preserving way as a subset of $S^{1}$, and quotient out complementary intervals to produce a circle $S_{\text {univ }}^{1}$. 
The images of $\overline{\mathcal{E}}^{ \pm}$in $S_{\text {univ }}^{1}$ are closed subsets. If we further quotient out complementary regions, we get quotient maps $\phi^{ \pm}: S_{\text {univ }}^{1} \rightarrow\left(S_{\text {univ }}^{1}\right)^{ \pm}$.

It remains to define the laminations $\Lambda_{\text {univ }}^{ \pm}$. For each leaf $l=\{a, b\}$ of $\Lambda^{+}$whose endpoints are in $\mathfrak{S}^{+}$, we can include $\mathfrak{S}^{+}$into $\overline{\mathfrak{S}}$ and map $\overline{\mathfrak{S}}$ to $S_{\text {univ }}^{1}$, and define $l_{\text {univ }}$ to be the leaf defined by the image of the endpoints. Then define $\Lambda_{\text {univ }}^{+}$to be the closure of the union of the leaves $l_{\text {univ }}$ obtained this way. Define $\Lambda_{\text {univ }}^{-}$similarly.

\subsection{Order trees}

To state the next corollary, we must recall the definition of an order tree.

Definition 5.9 An order tree is a set $T$ together with a collection $\mathcal{S}$ of linearly ordered subsets called segments, each with distinct least and greatest elements called the initial and final ends. If $\sigma$ is a segment, $-\sigma$ denotes the same subset with the reverse order, and is called the inverse of $\sigma$. The following conditions should be satisfied:

(1) If $\sigma \in \mathcal{S}$ then $-\sigma \in \mathcal{S}$.

(2) Any closed subinterval of a segment is a segment (if it has more than one element).

(3) Any two elements of $T$ can be joined by a finite sequence of segments $\sigma_{i}$ with the final end of $\sigma_{i}$ equal to the initial end of $\sigma_{i+1}$.

(4) Given a cyclic word $\sigma_{0} \sigma_{1} \cdots \sigma_{k-1}$ (subscripts $\bmod k$ ) with the final end of $\sigma_{i}$ equal to the initial end of $\sigma_{i+1}$, there is a subdivision of the $\sigma_{i}$ yielding a cyclic word $\rho_{0} \rho_{1} \cdots \rho_{n-1}$ which becomes the trivial word when adjacent inverse segments are cancelled.

(5) If $\sigma_{1}$ and $\sigma_{2}$ are segments whose intersection is a single element which is the final element of $\sigma_{1}$ and the initial element of $\sigma_{2}$ then $\sigma_{1} \cup \sigma_{2}$ is a segment.

If all the segments are homeomorphic to subintervals of $\mathbb{R}$ with their order topology, then $T$ is an $\mathbb{R}$-order tree.

An order tree is topologized by the usual order topology on segments. A lamination $\Lambda$ of a circle $S^{1}$ gives rise to a dual order tree $\Lambda^{*}$ as follows: think of $S^{1}$ as the ideal boundary of $\mathbb{H}^{2}$, and think of $\Lambda$ as the ideal points of leaves of a geodesic lamination $\Lambda_{g}$ of $\mathbb{H}^{2}$. The order tree $\Lambda^{*}$ is the quotient of the space of leaves of $\Lambda_{g}$ by the relation which identifies all boundary leaves of each complementary region. Each geodesic arc $\gamma \subset \mathbb{M}^{2}$ transverse to $\Lambda_{g}$ intersects $\Lambda_{g}$ in some closed subset, which inherits an order structure from $\gamma$; this defines the set $\mathcal{S}$. Notice that $\Lambda^{*}$ arising in this way is a Hausdorff order tree. 
Corollary 5.10 There are infinitely many closed hyperbolic 3-manifolds without quasigeodesic flows.

Proof In [5], Fenley shows that there are infinitely many hyperbolic surgeries $M_{i}$ on certain once-punctured torus bundles over the circle with the property that every action of $\pi_{1}\left(M_{i}\right)$ on an order tree has a global fixed point. Consider the manifold $M=M_{i}$. Suppose that $M$ admits a quasigeodesic flow $X$. By Theorem B, $\pi_{1}(M)$ acts faithfully on the order tree dual to the laminations $\Lambda_{\text {univ }}^{+}$. But by Fenley, this action globally fixes some point $p \in\left(\Lambda_{\text {univ }}^{+}\right)^{*}$.

It follows that under $\rho_{\text {univ }}^{+}$, the group $\pi_{1}(M)$ fixes the union of the leaves $\lambda_{i}$ which are dual to $p$. Such leaves correspond to the boundary leaves of the convex hull of the set of ends of some equivalence class $k$ of $\sim^{+}$, and therefore $\pi_{1}(M)$ must fix the corresponding point $e^{+}(k) \in S_{\infty}^{2}$. But the stabilizer of any point in $S_{\infty}^{2}$ is cyclic or trivial; this contradiction shows that $M$ does not admit a quasigeodesic flow, and the theorem is proved.

\section{Quasigeodesic flows and the Thurston norm}

In this section we demonstrate that the Thurston norm on a hyperbolic 3-manifold $M$ can be characterized in terms of the homotopy classes of quasigeodesic flows that $M$ supports. A basic reference for the Thurston norm is [26]. Another useful reference is [22].

\subsection{Circle bundles, plane bundles, and Euler classes}

Definition 6.1 Let $X$ be a $C^{1}$ flow on an oriented 3 -manifold $M$. Let $\xi$ be a complementary oriented 2-plane field, so that

$$
T X \oplus \xi=T M
$$

as oriented bundles. Let $e(\xi) \in H^{2}(M ; \mathbb{Z})$ be the obstruction to trivializing $\xi$ as a bundle. By abuse of notation, we denote the image of $e(\xi)$ in $H^{2}(M ; \mathbb{R})$ by $e(X)$.

The class $e(\xi)$ is an example of a characteristic class, and is called the Euler class of the bundle $\xi$.

Suppose $E$ is a bundle over a space $M$ with fiber homeomorphic to some fixed manifold $F$. There is a classifying space $\operatorname{BHomeo}(F)$ for $F$ bundles, and such bundles $E$ are classified by homotopy classes of maps $[M, \mathrm{BHomeo}(F)]$. A cohomology class $e \in H^{*}(\mathrm{BHomeo}(F))$ pulls back under such a homotopy class of map to a cohomology 
class on $M$, called a characteristic class. The Euler class of a circle bundle is an example of such a characteristic class. Both Homeo ${ }^{+}\left(S^{1}\right)$ and Homeo ${ }^{+}\left(\mathbb{R}^{2}\right)$ with the compact-open topology are homotopic to $S^{1}$, and therefore

$$
H^{*}\left(\mathrm{BHomeo}^{+}\left(S^{1}\right)\right)=H^{*}\left(\mathrm{BHomeo}^{+}\left(\mathbb{R}^{2}\right)\right)=\mathbb{Z}[e]
$$

where $e$ is free in dimension 2. The pullback of this class is called the Euler class of an oriented $S^{1}$ or $\mathbb{R}^{2}$ bundle over $M$. Note that since $\mathrm{BHomeo}^{+}\left(S^{1}\right)$ and BHomeo $^{+}\left(\mathbb{R}^{2}\right)$ are homotopic to $\mathbb{C P}^{\infty}$ which is a $K(\mathbb{Z}, 2)$, oriented plane or circle bundles are classified up to isomorphism by the Euler class. Note further that if $M$ is a hyperbolic 3 -manifold, $M$ is a $K(\pi, 1)$, and therefore the Euler class may be thought of as an element of group cohomology $H^{2}\left(\pi_{1}(M) ; \mathbb{Z}\right)$. See [14] or [17] for more details.

Associated to the representation $\rho_{\text {univ }}: \pi_{1}(M) \rightarrow \operatorname{Homeo}^{+}\left(S_{\text {univ }}^{1}\right)$ there is a (foliated) circle bundle $E_{\text {univ }}$ over $M$, defined by the usual Borel construction

$$
E_{\text {univ }}=\widetilde{M} \times S_{\text {univ }}^{1} /(m, \theta) \sim\left(\alpha(m), \rho_{\text {univ }}(\alpha)(\theta)\right)
$$

where $\alpha$ ranges over $\pi_{1}(M)$, and $m \in \widetilde{M}, \theta \in S_{\text {univ }}^{1}$ are arbitrary. Similarly, the natural action of $\pi_{1}(M)$ on $P_{X}$ defines a (foliated) $\mathbb{R}^{2}$ bundle $E_{P}$ over $M$, defined analogously.

For foliated $\mathbb{R}^{2}$ and $S^{1}$ bundles, we have the following algebraic definitions of the Euler class, by thinking of elements of $H^{2}(G ; \mathbb{Z})$ as central extensions:

Construction 6.2 Let $G$ be a subgroup of $\mathrm{Homeo}^{+}\left(S^{1}\right)$. Let $\widehat{G}$ denote the preimage of $G$ in the universal central extension $\mathrm{Homeo}^{+}\left(S^{1}\right)$ (the preimage of Homeo ${ }^{+}\left(S^{1}\right)$ in Homeo ${ }^{+}(\mathbb{R})$ under the universal covering map $\mathbb{R} \rightarrow S^{1}$ ). Then there is a central extension

$$
0 \rightarrow \mathbb{Z} \rightarrow \widehat{G} \rightarrow G \rightarrow 0
$$

The class of this extension gives the Euler class $\rho^{*}[e] \in H^{2}(G ; \mathbb{Z})$.

Construction 6.3 Let $G$ be a subgroup of Homeo ${ }^{+}\left(\mathbb{R}^{2}\right)$. Let $G_{\infty}$ denote the germ of $G$ at $\infty$. Let $A$ be a punctured disk neighborhood of $\infty$, and let $\widetilde{A}$ be the universal cover of $A$. Then there is a central extension

$$
0 \rightarrow \mathbb{Z} \rightarrow \widehat{G}_{\infty} \rightarrow G_{\infty} \rightarrow 0
$$

where $\widehat{G}_{\infty}$ denotes the subgroup of periodic germs of homeomorphisms of $\widetilde{A}$ which cover elements of $G_{\infty}$. The class of this extension pulls back by the natural restriction homomorphism $G \rightarrow G_{\infty}$ to give the Euler class $\rho^{*}[e] \in H^{2}(G ; \mathbb{Z})$. 
By considering the germs of equivalence classes of $\sim^{ \pm}$in $P_{X}$ at infinity, one sees that the Euler class of the foliated bundle $E_{P}$ is the obstruction to lifting the circular order on $\mathfrak{r}^{ \pm}$to a $\pi_{1}(M)$-invariant total order. It follows that the Euler classes of $E_{P}$ and $E_{\text {univ }}$ are equal.

See [2, Section 4.1] for a justification of this construction, and for more details.

Let $U \xi$ denote the circle bundle of unit vectors in $\xi$. Note that $U \xi$ and $\xi$ have the same Euler class as $S^{1}$ and $\mathbb{R}^{2}$ bundles respectively.

Lemma 6.4 The circle bundles $U \xi$ and $E_{\text {univ }}$ are isomorphic.

Proof At each point $x \in \widetilde{M}$, the plane $\xi(x)$ may be thought of as the tangent space at $x$ to the leaf space $P_{X}$ of $\widetilde{X_{\mathscr{Y}}}$. Note that the hypothesis that $X$ is $C^{1}$ implies that $P_{X}$ admits a natural $C^{1}$ structure. By exponentiating, one sees that $\xi$ and $E_{P}$ are isomorphic as plane bundles.

Moreover, by the discussion above, the Euler classes of $E_{P}$ and $E_{\text {univ }}$ are equal. The claim follows.

We will make use of a standard inequality known as the Milnor-Wood inequality, which is an inequality on the Euler class of a foliated circle bundle over a surface. See [16] and [28] for details.

Theorem 6.5 (Milnor-Wood) Let $E$ be an oriented foliated circle bundle over a closed orientable surface $S$. Then

$$
|e(E)[S]| \leq \max (0,-\chi(S)) .
$$

\subsection{The Thurston norm}

With Theorem 6.5 and Lemma 6.4 available to us, it is straightforward to prove the main result of this section.

Theorem $\mathbf{C}$ Let $M$ be a closed oriented hyperbolic 3-manifold. Then the convex hull of the set of Euler classes $e(X)$ as $X$ varies over the set of quasigeodesic flows on $M$ is exactly the unit ball of the dual Thurston norm on $H^{2}(M)$.

Proof Let $X$ be a quasigeodesic flow on $M$, and let $\xi$ be a complementary 2-plane field. Then by Lemma 6.4, $U \xi$ is isomorphic as a circle bundle to the foliated bundle $E_{\text {univ }}$. Let $\Sigma$ be a surface in $M$ which is Thurston norm minimizing for its homology class, so that $\|\Sigma\|_{T}=-\chi(\Sigma)$ where $\|\cdot\|_{T}$ denotes the Thurston norm. The bundle 
$E_{\text {univ }}$ restricts to a foliated circle bundle over $\Sigma$, so by the Milnor-Wood inequality Theorem 6.5, we have $|e(X)[\Sigma]| \leq\|\Sigma\|_{T}$; that is, $e(X)$ is contained in the unit ball of the Thurston norm.

Conversely, Gabai and Mosher [18] constructed a pseudo-Anosov flow almost transverse to any finite depth foliation. Fenley-Mosher [8] showed that these flows can all be taken to be quasigeodesic. Moreover, Gabai [9] constructed a finite depth foliation containing any given norm-minimizing surface as a compact leaf. This shows that for every $\Sigma$ as above, there is some quasigeodesic flow $X$ such that

$$
|e(X)[\Sigma]|=-\chi(\Sigma)=\|\Sigma\|_{T} .
$$

Now, such $X$ are not necessarily $C^{1}$, but they can be $C^{0}$ approximated by a $C^{1}$ flow $X^{\prime}$. Such $X^{\prime}$ is homotopic to $X$, and therefore has the same Euler class. Moreover, since $k$ quasigeodesity is a local property for any $k$, and since flowlines of $\widetilde{X_{\mathfrak{\mho}}^{\prime}}$ are arbitrarily close to flowlines of $\widetilde{X_{\mathscr{\mho}}}$ on compact subsets, it follows that $X^{\prime}$ is also quasigeodesic.

In particular, every extremal point in the unit ball of the dual Thurston norm can be realized, and therefore the conclusion follows.

As remarked in the introduction, this generalizes one of the main theorems of [19] and [20], for pseudo-Anosov quasigeodesic flows.

\section{References}

[1] D Calegari, The geometry of $\mathbf{R}$-covered foliations, Geom. Topol. 4 (2000) 457-515 MR1800151

[2] D Calegari, Circular groups, planar groups, and the Euler class, from: "Proceedings of the Casson Fest (Arkansas and Texas, 2003)", (C Gordon, Y Rieck, editors), Geom. Topol. Monogr. 7 (2004) 431-491 MR2172491

[3] D Calegari, N M Dunfield, Laminations and groups of homeomorphisms of the circle, Invent. Math. 152 (2003) 149-204 MR1965363

[4] J Cannon, W Thurston, Group invariant peano curves, preprint (1985)

[5] S R Fenley, Laminar free hyperbolic 3-manifolds arXiv:math.GT/0210482

[6] S R Fenley, Anosov flows in 3-manifolds, Ann. of Math. (2) 139 (1994) 79-115 MR1259365

[7] S R Fenley, Foliations, topology and geometry of 3-manifolds: R-covered foliations and transverse pseudo-Anosov flows, Comment. Math. Helv. 77 (2002) 415-490 MR1933786 
Universal circles for quasigeodesic flows

[8] S Fenley, L Mosher, Quasigeodesic flows in hyperbolic 3-manifolds, Topology 40 (2001) 503-537 MR1838993

[9] D Gabai, Foliations and the topology of 3-manifolds, J. Differential Geom. 18 (1983) 445-503 MR723813

[10] D Gabai, W H Kazez, The finiteness of the mapping class group for atoroidal 3-manifolds with genuine laminations, J. Differential Geom. 50 (1998) 123-127 MR1678485

[11] D Gabai, W H Kazez, Group negative curvature for 3-manifolds with genuine laminations, Geom. Topol. 2 (1998) 65-77 MR1619168

[12] D Gabai, U Oertel, Essential laminations in 3-manifolds, Ann. of Math. (2) 130 (1989) 41-73 MR1005607

[13] M Gromov, Hyperbolic groups, from: "Essays in group theory", Math. Sci. Res. Inst. Publ. 8, Springer, New York (1987) 75-263 MR919829

[14] D Husemoller, Fibre bundles, third edition, Graduate Texts in Mathematics 20, Springer, New York (1994) MR1249482

[15] M Kapovich, Hyperbolic manifolds and discrete groups, Progress in Mathematics 183, Birkhäuser, Boston (2001) MR1792613

[16] J Milnor, On the existence of a connection with curvature zero, Comment. Math. Helv. 32 (1958) 215-223 MR0095518

[17] J W Milnor, J D Stasheff, Characteristic classes, Annals of Mathematics Studies 76, Princeton University Press (1974) MR0440554

[18] L Mosher, Laminations and flows transverse to finite depth foliations, Part I: Branched surfaces and dynamics, preprint

[19] L Mosher, Dynamical systems and the homology norm of a 3-manifold. I. Efficient intersection of surfaces and flows, Duke Math. J. 65 (1992) 449-500 MR1154179

[20] L Mosher, Dynamical systems and the homology norm of a 3-manifold. II, Invent. Math. 107 (1992) 243-281 MR1144424

[21] L Mosher, Examples of quasi-geodesic flows on hyperbolic 3-manifolds, from: "Topology '90 (Columbus, OH, 1990)', Ohio State Univ. Math. Res. Inst. Publ. 1, de Gruyter, Berlin (1992) 227-241 MR1184414

[22] U Oertel, Homology branched surfaces: Thurston's norm on $\mathrm{H}_{2}\left(M^{3}\right)$, from: "Lowdimensional topology and Kleinian groups (Coventry/Durham, 1984)", London Math. Soc. Lecture Note Ser. 112, Cambridge Univ. Press (1986) 253-272 MR903869

[23] EH Spanier, Algebraic topology, McGraw-Hill Book Co., New York (1966) MR0210112

[24] WP Thurston, Hyperbolic Structures on 3-manifolds, II: Surface groups and 3manifolds which fiber over the circle arXiv:math.GT/9801045 
[25] WP Thurston, Three-manifolds, Foliations and Circles, II, preprint

[26] W P Thurston, A norm for the homology of 3-manifolds, Mem. Amer. Math. Soc. 59 (1986) i-vi and 99-130 MR823443

[27] R L Wilder, Topology of manifolds, American Mathematical Society Colloquium Publications 32, American Mathematical Society, Providence, R.I. (1979) MR598636 Reprint of 1963 edition

[28] J W Wood, Foliations on 3-manifolds, Ann. of Math. (2) 89 (1969) 336-358 MR0248873

[29] A Zeghib, Sur les feuilletages géodésiques continus des variétés hyperboliques, Invent. Math. 114 (1993) 193-206 MR1235023

Department of Mathematics, California Institute of Technology

Pasadena CA, 91125, USA

dannyc@its.caltech.edu

Proposed: David Gabai

Received: 15 June 2004

Seconded: Benson Farb, Walter Neumann

Revised: 10 September 2006 